\title{
Genetic screening of non-classic CAH females with hyperandrogenemia identifies a novel CYP11B1 gene mutation
}

\author{
Christos Shammas, ${ }^{1}$ Stefania Byrou, ${ }^{1}$ Marie M. Phelan, ${ }^{2}$ Meropi Toumba, ${ }^{3}$ \\ Charilaos Stylianou, ${ }^{4}$ Nicos Skordis, ${ }^{1}$ Vassos Neocleous, ${ }^{1}$ Leonidas A. Phylactou ${ }^{1}$
}

${ }^{1}$ Department of Molecular Genetics, Function \& Therapy, The Cyprus Institute of Neurology \& Genetics, Nicosia, Cyprus; ${ }^{2} \mathrm{NMR}$ Centre for Structural Biology, Institute of Integrative Biology, University of Liverpool, Liverpool, United Kingdom; ${ }^{3}$ Division of Pediatrics, lasis Hospital, Pafos, Cyprus; ${ }^{4}$ Pediatric Endocrinology, Pafos General Hospital, Cyprus; ${ }^{5}$ St George's, University of London Medical School at the University of Nicosia, Cyprus

\begin{abstract}
OBJECTIVE: Congenital adrenal hyperplasia (CAH) is an endocrine autosomal recessive disorder with various symptoms of diverse severity. Mild hyperandrogenemia is the most common clinical feature in non-classic CAH patients and $95 \%$ of the cases are identified by mutations in the CYP21A2 gene. In the present study, the second most common cause for non-classic CAH (NC-CAH), 11 -hydroxylase deficiency due to mutations in the CYP11B1 gene, is investigated. DESIGN: Screening of the $C Y P 21 A 2$ and $C Y P 11 B 1$ genes by direct sequencing was carried out for the detection of possible genetic defects in patients with suspected CAH. RESULTS: It was observed that $C Y P 11 B 1$ variants co-exist only in rare cases along with mutations in $C Y P 21 A 2$ in patients clinically diagnosed with $\mathrm{CAH}$. A total of $23 \mathrm{NC}-\mathrm{CAH}$ female patients out of 75 were identified with only one mutation in the CYP21A2 gene. The novel CYP11B1 gene mutation, p.Val484Asp, was identified in a patient with $\mathrm{CAH}$ in the heterozygous state. The structural characterization of the novel p.Val484Asp was found to likely cause distortion of the surrounding beta sheet and indirect destabilization of the cavity that occurs on the opposite face of the structural elements, leading to partial impairment of the enzymatic activity. CONCLUSIONS: CYP21 22 gene mutations are the most frequent genetic defects in cases of NC-CAH even when these patients are in the heterozygous state. These mutations have a diverse phenotype giving rise to a variable extent of cortisol synthesis impairment; it is also clear that CYP11B1 mutants are a rare type of defects causing CAH.
\end{abstract}

Key words: CYP11B1, Hyperandrogenemia, Non-classic CAH, PCOS, Premature adrenarche

Address for correspondence:

Leonidas A. Phylactou, Department of Molecular Genetics,

Function \& Therapy, The Cyprus Institute of Neurology \&

Genetics, 1683 Nicosia, Cyprus; E-mail: laphylac@cing.ac.cy

Received: 08-09-2015, Accepted: 22-12-2015

\section{INTRODUCTION}

Congenital adrenal hyperplasia $(\mathrm{CAH})$ is an endocrine autosomal recessive disorder characterized by the inability of the adrenal cortex to synthesize 
cortisol, which stimulates the secretion of ACTH. Elevated levels of ACTH cause adrenal hyperplasia due to the induction of adrenocortical growth. ${ }^{1}$ Steroid precursors are overproduced and deflected into the androgen pathway. ${ }^{2}$ Mutations in the CYP21A2 gene that cause 21-hydroxylase deficiency are the most common cause of the disease and account for approximately $95 \%$ of $\mathrm{CAH}$ patients. The second most common cause of CAH is $11 \beta$-hydroxylase deficiency due to mutations in the CYP11B1 gene. In some rare cases, $C A H$ can be due to mutations in the following genes: CYP11B2, CYP17, HSD $3 B 2$ and $S T A R^{3}$

$11 \beta$-hydroxylase isozymes encoded by the CYP11B1 and $C Y P 11 B 2$ genes $^{4}$ are members of the cytochrome 450 superfamily and are located on human chromosome $8 \mathrm{q}$, at a distance of approximately $40 \mathrm{~kb} .{ }^{4}$ Both genes have nine exons and are $95 \%$ identical in coding regions and $90 \%$ in introns, ${ }^{5}$ with a $440 \mathrm{bp}$ insertion in intron 5 of $C Y P 11 B 2$ being their biggest difference. ${ }^{2}$ $C Y P 11 B 1$ and $C Y P 11 B 2$ encode for proteins that share approximately $93 \%$ homology. ${ }^{2}$ The $C Y P 11 B 1$ gene encodes a protein with only $11 \beta$-hydroxylase activity. It catalyzes the synthesis of cortisol and is regulated by ACTH. ${ }^{6}$ CYP $11 B 2$ is responsible for aldosterone synthesis. It encodes a protein with 18 -hydroxylase and 18-oxydase activities and much lower activity of $11 \beta$-hydroxylase. Thus it is known as "aldosterone synthase" and is regulated by angiotensin II and $\mathrm{K}^{+}$ serum levels. ${ }^{5} \mathrm{CYP} 11 \mathrm{~B} 1$ mutations are responsible for $11 \beta$-hydroxylase deficiency ${ }^{6}$ with over 90 reported disease-causing CYP11B1 mutations. ${ }^{78}$ Recombination between these two homologous genes is common, resulting in a chimeric gene which usually encodes for a protein with aldosterone synthase activity regulated by ACTH, the CYP11B1 promoter. ${ }^{5}$ Nevertheless, the opposite case has been reported by Hampf et al concerning a chimeric gene encoding cortisol from the CYP11B2 promoter. $^{5}$ Mutations in the CYP11B1 gene cause $11 \beta$-hydroxylase deficiency, which is similar to 21-hydroxylase deficiency in the accumulation of steroid precursors due to stimulation of ACTH, and shift towards the androgen synthesis pathway (Table 1). ${ }^{9}$

CYP11B1 and CYP21A2 gene mutations give rise to hyperandrogenemia which is the most common clinical outcome for NC-CAH patients. Since CYP21A2 mutations account for $95 \%$ of $\mathrm{CAH}$ cases, it is not common practice for patients with hyperandrogenemia symptoms to undergo CYP11B1 screening. ${ }^{10,11}$ In this report, 75 patients with mild hyperandrogenemia identified with a single or no CYP21A2 gene mutations were evaluated further with screening for CYP11B1. The evaluation of CYP11B1 involvement in hyperandrogenemia can contribute to improved treatment strategies but also potentially reassesses the diagnostic criteria. In this report we examine the novel CYP11B1 gene mutation.

Table 1. A representation of the clinical features observed in patients with 21-OHD, 11-OHD and Aldosterone Synthase Deficiency. As can be observed by the table below there are no similar clinical characteristics between 11-OHD and Aldosterone Synthase Deficiency

21- hydroxylase deficiency

(CYP21A2)

Normal Cortisol

Hypertension

Hypotension

Hyperkalemia

Hypokalemia

Hyperandrogonemia

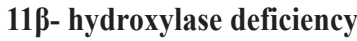

(CYP11B1)
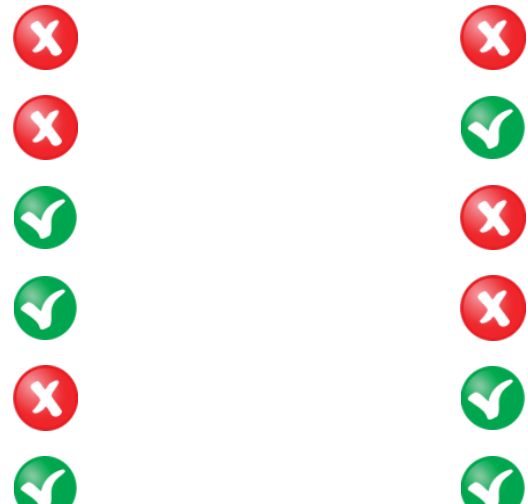

Aldosterone synthase deficiency (CYP11B2)

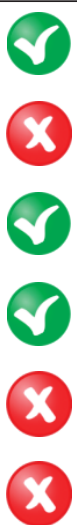




\section{SUBJECTS AND METHODS}

\section{Subjects}

Seventy-five female patients of Greek-Cypriot origin with mild symptoms of hyperandrogenemia participated in this study and written informed consent was obtained from all patients. Group A included 37 females who displayed a premature adrenarche phenotype and group B consisted of 38 individuals with polycystic ovary syndrome who were selected for sequencing.

\section{Hormonal assays}

Blood for hormone analyses was drawn after an overnight fast. Serum/plasma was either assayed immediately or frozen for later use. Standard recommended biochemical methods for measured parameters were employed.

\section{DNA amplification and sequence analysis.}

Genomic DNA was isolated from peripheral whole blood and the desired regions were amplified by polymerase chain reaction in reaction volumes of $20 \mu 1$ using 100ng genomic DNA. The CYP21A2 and $C Y P 11 B 1$ genes were analyzed according to a cascade strategy as previously described. ${ }^{12-14}$ The $C Y P 21 A 2$ gene primers $\mathrm{P} 1-\mathrm{P} 48^{13}$ were used to amplify the fragment containing the -370 bp CYP $21 A 2$ promoter, the 5 ' untranslated region of the $C Y P 21 A 2$ gene that is mainly located in the first 167 nucleotides upstream the ATG codon and the 3' untranslated region that is 536 nucleotides downstream from the TGA stop codon of the CYP21A2 gene. ${ }^{14}$ Meanwhile, the CYP11B1 region was amplified using 2 sets of primers, avoiding the homologous CYP11B2 gene, which were then sequenced using sequencing primers that cover exons $1-2,3-5$ and $6-9$, as previously described..$^{9,15}$

\section{MLPA analysis}

All samples underwent DNA analysis using the multiplex ligation-dependent probe amplification (MLPA) technique (MRC Holland, Amsterdam, The Netherlands). MLPA was employed to investigate in the CYP21A2 gene deleterious defects such as large gene deletions and large gene conversions which are harbored in the pseudogene $C Y P 21 P$ and can be transferred to the functional gene by homologous recombination.

\section{Structural analysis}

The characterization of the novel CYP11B1 mutation protein structure was based upon the UniProt (Universal Protein Resource) P15538 structure of CYP11B2, that shares $93 \%$ sequence identity, using the PROMALS3D construct alignment database. ${ }^{16,17}$ The CYP11B1 secondary structure elements were annotated with standard cytochrome P450 structural features [25a], as described in the alignment of CYP11A and CYP11B P450s. ${ }^{18}$ The structure of CYP11B1 both wild type (V484) and mutant (V484D) was modelled on crystal structures of CYP11B2; pdb ID 4FDH (CYP11B2 crystallized in complex with heme and fadrozole) and pdb ID 4DVQ (CYP11B2 crystallized in complex with heme and 21-hydroxyprogesterone).$^{19} \mathrm{CYP} 11 \mathrm{~B} 1$ was modelled using the protein-BLAST alignment [27a] with CYP11B2 using both 4FDH and 4DVQ structures as a template via modelling software Modeller version 9.11.20,21 Of the twenty model structures generated the model with the lowest objective score was selected and used for structural analysis. The mutant V484D was modelled in the same manner. The structural analysis was carried out using the PyMOL Molecular Graphics System, Version 1.3, Schrödinger, LLC, for structure visualization and residue conformation assessment. The residues in close proximity to the heme and steroid moiety were identified using $\mathrm{PIC}^{22}$ LigPlot $^{23}$ and EBI pdb analysis suite PDBsum. ${ }^{24}$

\section{Statistical analysis}

A comparison was carried out between the female patients of group A with PA who were heterozygous for CYP21A2 mutations and the patients of group $\mathrm{B}$ with polycystic ovary syndrome (PCOS) using a chi-square test. A significance level of 0.05 was used to establish statistical significance. The statistical analyses were performed using SAS software Version 9.2, SAS Institute Inc., Cary, NC, USA.

\section{RESULTS}

\section{Genetic screening}

Of the 75 female patients selected belonging to group A with PA and group B with PCOS, 23 (30.66\%) were found heterozygous for CYP $21 A 2$ gene mutations, whereas the remaining $52(69.33 \%)$ 
had no CYP21A2 mutations (supplementary Table 1). Sixteen $(43.2 \%)$ out of the 37 patients of group A with PA were found heterozygous for CYP21A2 mutations vs 7/38 (18.4\%) patients of group B with PCOS (pvalue $<0.020)$. Genetic screening for $C Y P 11 B 1$ gene mutations revealed one heterozygous patient with the novel mutation, p.Val484Asp. One patient was identified with the known $C Y P 11 B 1$ variant, p.Arg43Gln, and the severe CYP21A2 mutation, p.Gln318Ter. The p.Gln318Ter mutation has a strong association with a trimodular haplotype which is non-disease causing. ${ }^{25}$ Therefore, the p.Gln318Ter was verified, in addition to Sanger sequencing, using MLPA analysis.

Patient 1 is a female adolescent of Greek/Cypriot origin who was initially presented at the age of $71 / 2$ years with obesity (BMI: $+2.23 \mathrm{SDS}$ ) and with premature adrenarche. She had pubic hair at Tanner stage 3 and axilary hair at Tanner stage 2 as well as body odor. Her bone age was advanced by 2 years according to the Greulich and Pyle method. She had normal blood pressure for age and height. Her fasting glucose, electrolytes, kidney function, lipid profile, liver and thyroid function tests were within the normal levels for age. Her baseline cortisol, insulin, 17-hydroxyprogesterone (17-OHP), androstenedione and DHEA-S were at normal levels, ACTH levels were $42 \mathrm{pg} / \mathrm{ml}$ and 11-deoxycortisol levels $105 \mathrm{ng} / \mathrm{dl}$, i.e. both within the normal range for age. She started menstruating at the age of 11 years and, at this point, her BMI was dramatically increased. At the age of 12 she was obese with BMI +2.8 SDS and she presented with acanthosis nigricans, acne and oligomenorrhea. She had no hypertension. Her baseline 17-OHP was slightly elevated $(1.8 \mathrm{ng} / \mathrm{ml}$, range: $0.2-1.2 \mathrm{ng} / \mathrm{ml})$ and she developed insulin resistance, evaluated by homeostatic model assessment (HOMA $>2$ ). The rest of her baseline hormonal and biochemical tests were within the normal ranges. She refused ACTH and OGTT tests. She was advised to start dieting and exercising and she was commenced on treatment with metformin. This female adolescent is also a carrier of the severe p.Gln318Ter mutation in the CYP21A2 gene and, according to the bibliography, this mutation in heterozygosity could cause hyperandrogenemia. It seems that these mutations coexist and act synergistically in the premature adrenarche phenotype.

The patient with the novel CYP11B1 mutation in heterozygosity, Patient 2, is a Greek-Cypriot female who initially presented at the age of 18 years due to oligomenorrhea and androgenic alopecia. She mentioned early adrenarche at the age of 8 years and menarche at the age of 12 years. She had had irregular menses since then. On the day of presentation her BMI was $27 \mathrm{~kg} / \mathrm{m}^{2}$ and she had no hypertension. She was diagnosed with PCOS according to the Androgen Excess Society criteria. ${ }^{26}$ She had normal levels of electrolytes and normal kidney and liver function. Her fasting glucose, insulin, morning cortisol and 17-OHP levels were unremarkable (17OHP: $0.5 \mathrm{ng} /$ $\mathrm{ml} / 1$, range:0.2-1.2). She had elevated delta 4-androstenedione $(\Delta 4)(5.4 \mathrm{ng} / \mathrm{ml}$, range:0.3-3.3) and slightly elevated DHEA-S (dehydroepiandrosterone -sulphate) (12.10 $\mu \mathrm{mol} / \mathrm{L}$, range:0.94-11.67), measured on the $4^{\text {th }}$ day of a spontaneous menstrual cycle. She was treated with a hormonal combination contraceptive pill. Genetic screening for both $C Y P 21 A 2$ and $C Y P 11 B 1$ mutations revealed a novel mutation on the $C Y P 11 B 1$ gene. This mutation is located at codon 484 and replaces a non-polar hydrophobic amino acid (valine) for a positively charged polar amino acid (aspartic acid) in one of the two alleles.

\section{Structural characterization}

CYP11B proteins are steroid binding enzymes whose activity is dependent on colocalization of a heme molecule. Predominantly helical in composition, the helices arrange to form a deep cavity around the heme molecule and adjacently placed steroid substrate. ${ }^{18}$

As previously discussed, the $C Y P 11 B 1$ and $C Y$ $P 11 B 2$ genes are arranged tandemly and encode proteins of $93 \%$ identity; nevertheless, the functions of these highly homologous proteins are distinct. ${ }^{19}$ This distinction between CYP11B isoforms arises from the binding of different steroid substrates.

The novel mutation identified in Patient 2 is not located within the binding cavity but is surface facing arranged on one of the few beta strands. The apo form of the amino acid is a valine which is conserved between CYP11B1 and CYP11B2, as are many of the amino acid networks in close proximity to this residue in the crystal structure(s) 4FDH and 4DVQ. These residues are located on the same beta strand $\beta 4-1$ (K482, M483 and Y485), adjacent beta strand $\beta 4-2$ (R490, P491 and S/G492), the beta hairpin loop 
(S486) and one residue from a loop between helices $\alpha \mathrm{A}^{\prime}$ and $\alpha \mathrm{A}$ at position E62. Of these compactly arranged residues only one is varied between $\mathrm{C} 11 \mathrm{~B} 1$ and C11B2, that is, G492 and S492, respectively (Figure 1).

Valine 484 is localized on the surface face of a beta strand, $\beta 4-1$, along with K482, M483 and Y485 amino acids and adjacent to beta strand $\beta 4-2$ and in close proximity to the $\alpha \mathrm{A}^{\prime}$ and $\alpha \mathrm{A}$ loops. This region is not considered to have any steroid binding role but is within Van der Waals contact (approximately $5 \AA$ proximity $^{30}$ ) to the hydrophobic residues $\mathrm{F} 487$ and I488 that is conserved between both CYP11B1 and CYP11B2 (Figure 1). It is apparent that the model we have produced of CYP11B1 based on the CYP11B2-Heme-Fadrozole complex contains the same hydrophobic-rich binding cavity. An electrostatic clash between the acidic aspartate at position 484 and glutamate at position 62 may indirectly cause destabilization of the binding cavity on the opposite face of the structural elements. It is postulated that the repulsion between electronegative oxygen moieties of D484 and E62 may cause a distortion on the surrounding beta sheet which includes residues F487 and I488 of the steroid binding pocket, thus indirectly destabilizing the binding of the steroid to CYP11B.

\section{DISCUSSION}

$\mathrm{CAH}$ is a condition with diverse severity of symptoms that can be explained by various $\mathrm{CAH}$ causing genes. However, most importantly there are different types of mutations in the same gene that can cause from full to partial impairment of enzymatic activity. The combination of different types of CAH mutations affects the level of clinical expression of the disease. ${ }^{27}$ Patient 1 did not have elevated 17-OHP levels as observed with other heterozygous NC-CAH cases with a single p.Gln318Ter mutation; ${ }^{2,8-29}$ on the other hand, a higher than normal 17-OHP level might have been observed after ACTH stimulation. The p.Arg43Gln variant replaced a positively charged polar amino acid with an uncharged polar glutamine. This variation was initially characterized as non-causative, ${ }^{5}$ but later on Barr et al and Parajes et al characterized this variant as causative with approximately $10-50 \%$ reduction of efficiency, leading to a mild phenotype. ${ }^{30-32}$ Although

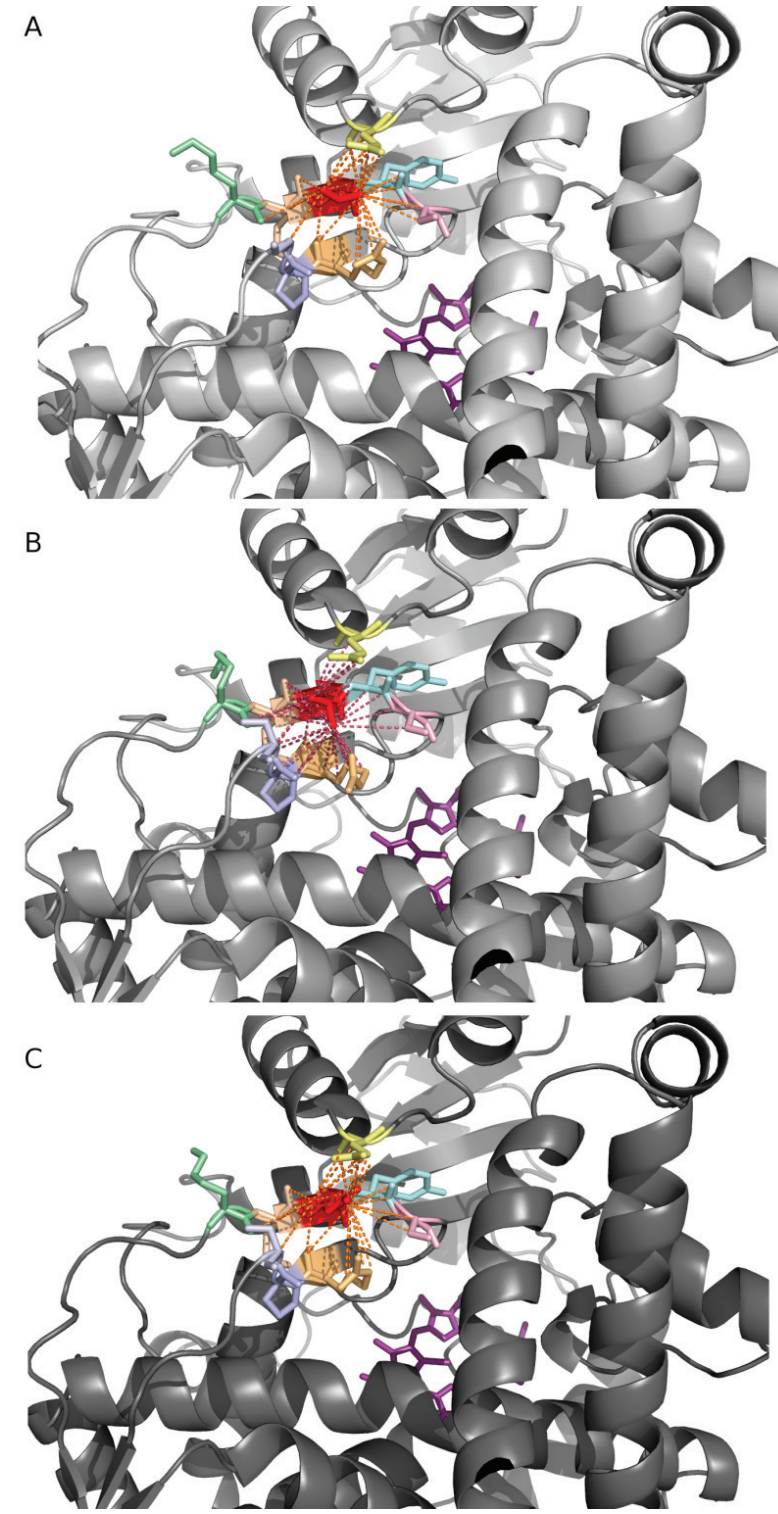

Figure 1. A) Wild Type crystal structure of CYP11B2. B) Wild type homology model of CYP11B1. C) Mutant V484D homology model of CYP11B1. Residue 484 valine in A) and B) and aspartate in $\mathrm{C}$ ) shown in red with residues in close proximity ( $5 \AA$ or less) indicated by dashed lines: E62, yellow; K482, green; M483, salmon; Y485, cyan; S486, pink; R490, orange; P491, mauve; and S492 (CYP11B2) or G492 (CYP11B1), blue. The crystal structure is based on 4FDH. Based on the crystallized structure of CYP11B2 S486 is part of the beta hairpin loop and shares interatomic interactions with its neighboring residues. Residues K482, M483 and Y485 are located on the same beta strand $\beta 4-1$ but adjacent to the beta strand $\beta 4-2$ with residues R490, P491 and S/G492 and the beta hairpin loop that consist the S486 and one residue from a loop between helices $\alpha \mathrm{A}^{\prime}$ and $\alpha \mathrm{A}$ at position $\mathrm{E} 62$. Within these compactly arranged residues of these beta hairpin loop structures, G492 of CYP11B1 is replaced with S492 of CYP11B2. 
the different physical properties of the amino acids are a major reason for the changes in enzymatic activity, the short distance of a variation to an active site of the enzyme can also be causative. In 2001, Belkina et al studied the three-dimensional structures of the $C Y P 11 B 1$ and CYP11B2 genes and proposed the I helix of $11 \beta$-hydroxylase as the putative active site. ${ }^{33}$ Since the variant p.Arg43Gln is located within the $\mathrm{N}$-term ${ }^{31}$ and not within the helix I, Barr et al assumed that this specific variant disturbs a substrate recognition site or influences the flexibility of the enzyme and disrupts the substrate-active site combination. ${ }^{30}$ It should also be noted that p.Arg43Gln changes a basic arginine amino acid into a neutral polar glutamine amino acid, suggesting functional protein impairment.

Patient 2 was found to be a symptomatic carrier of the novel p.Val484Asp mutation and it can be assumed that this mutation contributes to the partial enzyme impairment. The specific mutation exchanges a nonpolar valine hydrophobic amino acid for a positively charged polar aspartic acid amino acid at codon region 484, causing PCOS. Neighboring codons Tyr485 and Ile488 are parts of the active CYP $11 B 1$ gene site. ${ }^{33}$ It can therefore be predicted that mutation $\mathrm{p}$.Val484Asp affects the local conformation and disturbs the efficiency of $11 \beta$-hydroxylase thereby contributing to the patient's phenotype. Interestingly, in silico mutation analyses that were performed initially using Provean software ${ }^{34}$ predicted a deleterious effect of the p.Val484Asp alteration on CYP11B1 function (Table 2).

Genetic screening for CYP11B1 mutations in 75 Greek-Cypriot female patients with mild symptoms of hyperandrogenemia included: Group A with 37 females who displayed a premature adrenarche phenotype, and group B with 38 females with polycystic ovary syndrome all of whom had been previously examined for mutations in the CYP21A2 gene. Of the 75 patients, 23 were found to be heterozygotes, whereas the remaining 52 had no CYP21A2 mutations. The

Table 2. Provean function prediction of the $\mathrm{p}$.Val484Asp variation in CYP11B1 $1^{39}$

\begin{tabular}{lcc}
\hline Variant & PROVEAN score & Prediction (cutoff= -2.5) \\
\hline p.Val484Asp & -3.777 & Deleterious \\
\hline
\end{tabular}

finding from our previous study that demonstrating the frequency of the underlying genetic CYP21A2 defects in the Cypriot population to be one of highest ever reported and with an allelic frequency of 1:10 corroborated the observed heterozygotes of the present study. ${ }^{35}$ Several studies have demonstrated CYP11B1 gene defects to be rare, therefore, it is not surprising that of the patients tested in the present study only three NC-CAH patients were identified with $C Y P 11 B 1$ gene mutations. ${ }^{3,8,36}$

The detection of only two carriers of $11 \beta$-hydroxylase deficiency mutations among female patients with mild hyperandrogenemia is consistent with other studies and demonstrates that CYP11B1 gene mutations account for a small minority of cases in females with hyperandrogenism. ${ }^{37}$ The novel $p$.Val484Asp mutation was found to cause a distortion of the surrounding beta sheet and also to result indirectly in destabilization of the binding cavity that occurs on the opposite face of the structural elements, leading to partial impairment of the enzymatic activity. Reporting novel mutations and even combinations are of great importance as such information can enable a clearer diagnosis and contributes to a better treatment modality. Understanding the extent of cortisol synthesis impairment caused by specific mutations but also due to a combination of mutations is essential for correct diagnosis, prognosis and treatment. In this report we examine the deleterious effect of a novel CYP11B1 mutation and, in addition, how heterozygosity in $C Y P 21 A 2$ as well as cases with no identified mutations could lead to a NC-CAH phenotype similar to that observed in the most prevalent type of the disorder with two identified mutations.

\section{ACKNOWLEDGMENTS}

This work was supported by the A.G. Leventis Foundation.

\section{CONFLICT OF INTEREST}

The authors do not have any conflict of interest.

\section{REFERENCES}

1. Falhammar H, Thoren M, 2012 Clinical outcomes in the management of congenital adrenal hyperplasia. 
Endocrine 41: 355-373.

2. Mornet E, Dupont J, Vitek A, White PC, 1989 Characterization of two genes encoding human steroid 11 beta-hydroxylase (P-450(11) beta). J Biol Chem 264: 20961-20967.

3. White PC, Speiser PW, 2000 Congenital adrenal hyperplasia due to 21-hydroxylase deficiency. Endocr Rev 21: 245-291.

4. White PC, Rainey WE, 2005 Editorial: polymorphisms in CYP11B genes and 11-hydroxylase activity. J Clin Endocrinol Metab 90: 1252-1255.

5. Hampf M, Dao NT, Hoan NT, Bernhardt R, 2001 Unequal crossing-over between aldosterone synthase and 11 beta-hydroxylase genes causes congenital adrenal hyperplasia. J Clin Endocrinol Metab 86: 4445-4452.

6. Li-Qiang Zhao SH, Hao-Ming Tian, 2008 Progress in molecular-genetic studies on congenital adrenal hyperplasia due to $11 \beta$-hydroxylase deficiency. World J Pediatr 4: 85-90.

7. Nimkarn S, Lin-Su K, New MI, 2011 Steroid 21 hydroxylase deficiency congenital adrenal hyperplasia. Pediatr Clin North Am 58: 1281-1300, xii.

8. Parajes S, Loidi L, Reisch N, et al, 2010 Functional consequences of seven novel mutations in the CYP11B1 gene: four mutations associated with nonclassic and three mutations causing classic 11 \{beta\}-hydroxylase deficiency. J Clin Endocrinol Metab 95: 779-788.

9. White PC, Dupont J, New MI, et al, 1991 A mutation in CYP11B1 (Arg-448----His) associated with steroid 11 beta-hydroxylase deficiency in Jews of Moroccan origin. J Clin Invest 87: 1664-1667.

10. Unluhizarci K, Kula M, Dundar M, et al, 2010 The prevalence of non-classic adrenal hyperplasia among Turkish women with hyperandrogenism. Gynecol Endocrinol 26: 139-143.

11. Moran C, Knochenhauer ES, Azziz R, 1998 Non-classic adrenal hyperplasia in hyperandrogenism: a reappraisal. J Endocrinol Invest 21: 707-720.

12. Shammas C, Neocleous V, Toumba M, et al, 2012 Overview of genetic defects in endocrinopathies in the island of Cyprus; evidence of a founder effect. Genet Test Mol Biomarkers 16: 1073-1079.

13. Speiser PW, Knochenhauer ES, Dewailly D, et al, 2000 A multicenter study of women with nonclassical congenital adrenal hyperplasia: relationship between genotype and phenotype. Mol Genet Metab 71: 527-534.

14. Wedell A, Luthman H, 1993 Steroid 21-hydroxylase deficiency: two additional mutations in salt-wasting disease and rapid screening of disease-causing mutations. Hum Mol Genet 2: 499-504.

15. Nguyen HH, Nguyen TH, Vu CD, et al, 2012 Novel homozygous p.Y395X mutation in the CYP11B1 gene found in a Vietnamese patient with 11 beta-hydroxylase deficiency. Gene 509: 295-297.

16. Pei J, Grishin NV, 2007 PROMALS: towards accurate multiple sequence alignments of distantly related pro- teins. Bioinformatics 23: 802-808.

17. Pei J, Kim BH, Grishin NV, 2008 PROMALS3D: a tool for multiple protein sequence and structure alignments. Nucleic Acids Res 36: 2295-2300.

18. Strushkevich N, MacKenzie F, Cherkesova T, et al, 2011 Structural basis for pregnenolone biosynthesis by the mitochondrial monooxygenase system. Proc Natl Acad Sci U S A 108: 10139-10143.

19. Strushkevich N, Gilep AA, Shen L, et al, 2013 Structural insights into aldosterone synthase substrate specificity and targeted inhibition. Mol Endocrinol 27: 315-324.

20. Eswar N, Webb B, Marti-Renom MA, et al, 2007 Comparative protein structure modeling using MODELLER. Curr Protoc Protein Sci Chapter 2: Unit 29.

21. Eswar N, Eramian D, Webb B, Shen MY, Sali A, 2008 Protein structure modeling with MODELLER. Methods Mol Biol 426: 145-159.

22. Tina KG, Bhadra R, Srinivasan N, 2007 PIC: Protein Interactions Calculator. Nucleic Acids Res 35: W473476.

23. Wallace AC, Laskowski RA, Thornton JM, 1995 LIGPLOT: a program to generate schematic diagrams of protein-ligand interactions. Protein Eng 8: 127-134.

24. de Beer TA, Berka K, Thornton JM, Laskowski RA, 2014 PDBsum additions. Nucleic Acids Res 42: D292296.

25. Parajes S, Quinteiro C, Dominguez F, Loidi L, 2008 High frequency of copy number variations and sequence variants at CYP21A2 locus: implication for the genetic diagnosis of 21-hydroxylase deficiency. PLoS One 3: e2138.

26. Escobar-Morreale HF, Carmina E, Dewailly D, et al, 2012 Epidemiology, diagnosis and management of hirsutism: a consensus statement by the Androgen Excess and Polycystic Ovary Syndrome Society. Hum Reprod Update 18: 146-170.

27. Skordis N, Shammas C, Efstathiou E, et al, 2011 Endocrine profile and phenotype-genotype correlation in unrelated patients with non-classical congenital adrenal hyperplasia. Clin Biochem 44: 959-963.

28. Salinas Vert I, Audi Parera L, Granada Ybern ML, et al, 1998 [Defects of adrenal steroidogenesis in patients with hirsutism]. Med Clin (Barc) 110: 171-176.

29. Speiser PW, Azziz R, Baskin LS, et al, 2010 Congenital adrenal hyperplasia due to steroid 21-hydroxylase deficiency: an Endocrine Society clinical practice guideline. J Clin Endocrinol Metab 95: 4133-4160.

30. Barr M, MacKenzie SM, Wilkinson DM, et al, 2006 Functional effects of genetic variants in the 11 betahydroxylase (CYP11B1) gene. Clinical Endocrinol 65: 816-825.

31. Parajes S, Loidi L, Reisch N, et al, 2010 Functional consequences of seven novel mutations in the CYP11B1 gene: four mutations associated with nonclassic and three mutations causing classic 11 \{beta\}-hydroxylase deficiency. J Clin Endocrinol Metab 95: 779-788. 
32. Barr M, MacKenzie SM, Wilkinson DM, et al, 2006 Functional effects of genetic variants in the 11 betahydroxylase (CYP11B1) gene. Clin Endocrinol (Oxf) 65: 816-825.

33. Belkina NV, Lisurek M, Ivanov AS, Bernhardt R, 2001 Modelling of three-dimensional structures of cytochromes P450 11B1 and 11B2. J Inorg Biochem 87: 197-207.

34. http://provean.jcvi.org/.

35. Phedonos AA, Shammas C, Skordis N, et al, 2013
High carrier frequency of 21-hydroxylase deficiency in Cyprus. Clin Genet 84: 585-588.

36. White PC, Rainey WE, 2005 Editorial: polymorphisms in CYP11B genes and 11-hydroxylase activity. J Clin Endocrinol Metab 90: 1252-1255.

37. Joehrer K, Geley S, Strasser-Wozak EM, et al, 1997 CYP11B1 mutations causing non-classic adrenal hyperplasia due to 11 beta-hydroxylase deficiency. Hum Mol Genet 6: 1829-1834. 